C 2021, American Psychological Association.

This paper is not the copy of record and may not exactly replicate the final, authoritative version of the article. Please do not copy or cite without authors' permission. The final article will be available, upon publication, via its DOI: 10.1037/rev0000316

\title{
Assessing the 'Paradox' of Converging Evidence by Modeling the Joint \\ Distribution of Individual Differences: Comment on Davis-Stober and \\ Regenwetter (2019)
}

\author{
Daniel W. Heck \\ Philipps University of Marburg
}

Version: June 4, 2021

\section{Author Note}

Data and R scripts for the analyses are available at the Open Science Framework (https://osf.io/7fk49/).

The present work was presented at the $62^{\text {nd }}$ Conference of Experimental Psychologists (Virtual TeaP, 2021). A preprint was uploaded to PsyArXiv and ResearchGate for timely dissemination (https://psyarxiv.com/ca8z4/).

This work was supported by the research training group Statistical Modeling in Psychology, funded by the German Research Foundation (DFG; grant GRK 2277) and by the Heidelberg Academy of Sciences and Humanities (WIN project Shared Data Sources).

The author is thankful to Clint Davis-Stober, Mike Regenwetter, and Henrik Singmann for clarifications and feedback on earlier versions of the manuscript.

Correspondence concerning this article should be addressed to Daniel W. Heck, Department of Psychology, Philipps University of Marburg, Gutenbergstraße 18, Room 01058, D-35032 Marburg, Germany. E-mail: dheck@uni-marburg.de. 


\begin{abstract}
Davis-Stober and Regenwetter (2019; D\&R) showed that even when all predictions of a theory hold in separate studies, not even a single individual may be described by all predictions jointly. To illustrate this 'paradox' of converging evidence, $\mathrm{D} \& \mathrm{R}$ derived upper and lower bounds on the proportion of individuals for whom all predictions of a theory hold. These bounds reflect extreme positive and negative stochastic dependence of individual differences across predictions. However, psychological theories often make more specific assumptions such as true individual differences being independent or positively correlated (e.g., due to a common underlying trait). Based on this psychometric perspective, I extend D\&R's conceptual framework by developing a multivariate normal model of individual effects. Assuming perfect consistency (i.e., a correlation of one) of individual effects across predictions, the proportion of individuals described by all predictions of a theory is identical to D\&R's upper bound. The proportion drops substantially when assuming independence of individual effects. However, irrespective of the assumed correlation structure, the multivariate normal model implies a lower bound that is strictly above D\&R's lower bound if a theory makes at least three predictions. Hence, the scope of a theory can be improved by specifying whether individual effects are assumed to show a certain level of consistency across predictions (similar to a trait) or whether they are statistically independent (similar to a state).
\end{abstract}

Keywords: Theoretical scope, theory development, heterogeneity, psychometrics, effect size. 


\section{Assessing the 'Paradox' of Converging Evidence by Modeling the Joint Distribution of Individual Differences: Comment on Davis-Stober and Regenwetter (2019)}

Davis-Stober and Regenwetter (2019, D\&R) investigated the implications of assuming that individuals differ in psychological constructs. For this purpose, they viewed a psychological theory as the logical conjunction of all its predictions (e.g., "the effects X, Y, and Z hold jointly"). The 'paradox' of converging evidence states that, even when all predictions are supported on average in separate studies, the proportion of individuals described by all predictions of a theory can be very small and may even be zero. With each additional prediction that is supported on average, it becomes more likely to find individuals who are exceptions to the theory. Hence, it is logically invalid to conclude from the fact that all predictions of a theory hold on the population level, that the theory holds for all or at least some individuals.

The 'paradox' of converging evidence concerns the specification and scope of any theory that assumes true individual differences in psychological constructs. Notably, it also applies when assuming that the average population effect sizes of all predictions are known perfectly. Hence, the issue cannot simply be resolved by improving statistical methods or by collecting more data in large-scale replication studies. Instead, scholars need to specify conceptually whether a theory and all its predictions are assumed to hold for all individuals in a population (for a detailed discussion, see D\&R). The present comment shows that additional assumptions about the joint distribution and the consistency of individual differences across predictions (i.e., about the correlation of individual effects) can increase the scope of a theory. When assuming true individual differences, scholars should specify whether individual effects show a certain level of consistency (similar to a trait) or whether they are statistically independent (similar to a state). By modeling the joint distribution of individual differences, I address D\&R's call that "future work [should] dig more deeply into how different predictions are interrelated" (p. 877). 


\section{Proportion of Individuals Described by a Theory}

D\&R focused on psychological theories that assume the existence of individual differences. More precisely, they investigated the consequences of assuming that each prediction of a theory holds only for some but not all individuals in the population. In this case, the question arises what can be concluded about the proportion $p$ of individuals in the population who are described by all predictions of the theory. D\&R showed that the proportion $p$ cannot assume arbitrary values but is restricted to lie between an upper and a lower bound.

The derivation of these bounds requires assumptions about the proportion $p_{i}$ of individuals for whom the $i^{\text {th }}$ prediction holds. The proportion $p$ of individuals described by all $k$ predictions of the theory must then satisfy the inequality

$$
\underbrace{\max \left\{0, p_{1}+\cdots+p_{k}-(k-1)\right\}}_{\text {lower bound }} \leq p \leq \underbrace{\min \left\{p_{1}, \ldots, p_{k}\right\}}_{\text {upper bound }}
$$

The upper bound follows directly by considering the prediction $i$ that describes the smallest proportion $p_{i}^{\min }$ of individuals in the population. In the best-case scenario, all other predictions also hold for these same individuals in which case the upper bound is tight (i.e., $p=p_{i}^{\min }$ ). The lower bound is derived by considering the worst-case scenario in which each individual violates a different prediction. For instance, some individuals may be described only by the first prediction but not by the second, whereas others may be described only by the second prediction but not by the third, and so on. When adding more and more predictions in such a way, the lower bound decreases monotonically. Based on this 'packing argument,' it may be possible construct a scenario in which not even a single individual is described by all predictions of a theory (i.e., $p=0$ ).

The distribution-free bounds in Equation (1) on the probability of the conjunction of a set of events are known as Fréchet inequalities in probabilistic logic (Fréchet, 1951) and as Fréchet-Hoeffding bounds in copula theory (Colonius, 2016). Fréchet also derived upper and lower bounds on the probability of the disjunction of a set of events. However, since scientific theories of the form "at least one of the effects X, Y, or Z holds" are 
hardly falsifiable (Davis-Stober \& Regenwetter, 2019; Popper, 2002), the case of disjunctions is not relevant for the present discussion. More importantly, Fréchet's upper and lower bounds on the probability of a conjunction reflect the most extreme stochastic dependence that is possible according to probability theory (Lee \& Ahn, 2014). The bounds require assumptions only about the marginal distributions of a set of random variables (here, about the proportions $p_{i}$ ) but not about their multivariate joint distribution. With respect to the discussion about the scope of psychological theories, this means that D\&R's bounds do not require assumptions about the stochastic dependence of individual differences across predictions.

\section{Average Population Effect Size}

D\&R applied the upper and lower bounds on the probability of a conjunction to study the scope of psychological theories. For this purpose, it is necessary to obtain an estimate of the proportion $p_{i}$ of individuals described by the $i^{\text {th }}$ prediction of a theory. Let us assume that the evidence for the predictions of a theory can be described in terms of standardized mean differences (i.e., Cohen's $d$ ). The following derivation assumes an ideal hypothetical situation in which the true population effect sizes are accurately known without sampling error (see D\&R, p. 865). On the population level, Cohen's $d$ is defined as $d=\left(\mu_{T}-\mu_{C}\right) / \sigma$, the difference between the population means in two groups (e.g., the treatment and the control condition) standardized by the within-group standard deviation $\sigma$.

To obtain the lower and upper bounds in Equation (1), it is necessary to transform Cohen's $d$ into an estimate of the proportion $p_{i}$. For this purpose, D\&R proposed a univariate normal model which is illustrated for three predictions in Figure 1. Drawing on classical test theory (Lord \& Novick, 1968), the model partitions the observed values in the treatment and control condition $\left(X_{T}\right.$ and $\left.X_{C}\right)$ into the true scores of an individual $\left(H_{T}\right.$ and $\left.H_{C}\right)$ and the corresponding measurement errors $\left(E_{T}\right.$ and $\left.E_{C}\right)$. Thereby, the model implies a decomposition of the within-group variance $\sigma^{2}$ which appears in the denominator of Cohen's $d$. More precisely, the within-group variance is 


\section{Figure 1}

Univariate Normal Model by Davis-Stober and Regenwetter (2019).

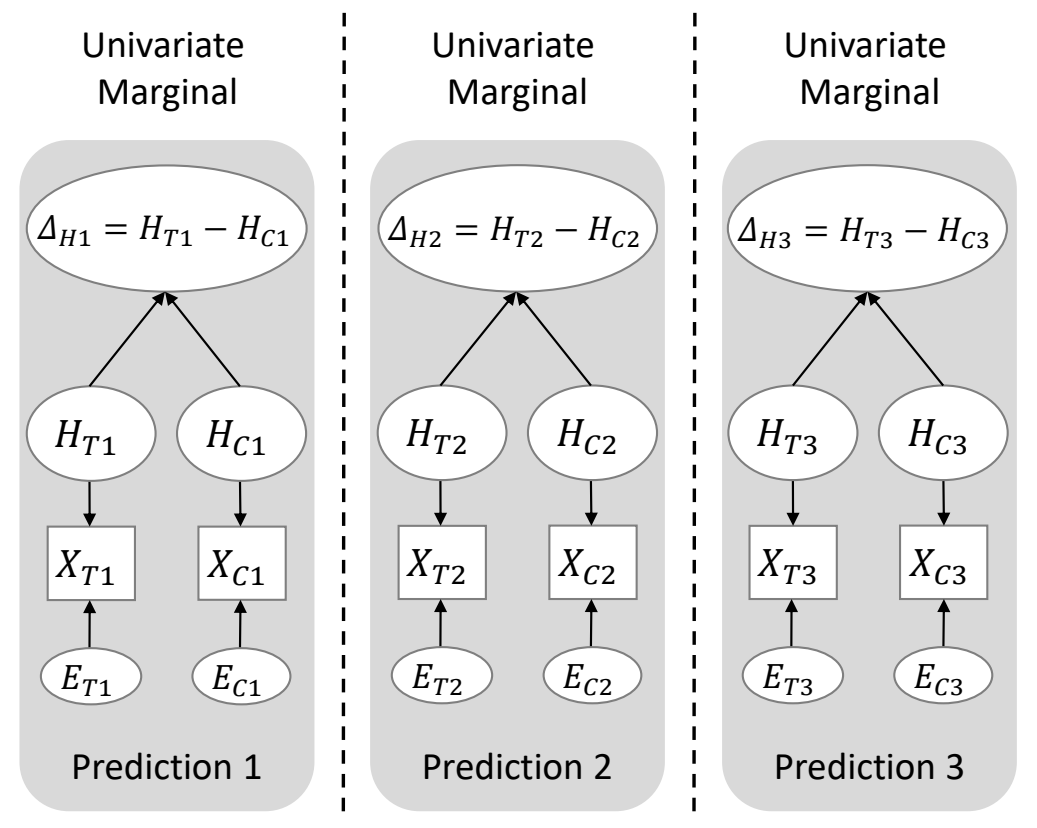

Note. D\&R's modeling approach does not assume a joint distribution of true individual effects $\Delta_{H}$ across predictions but only specifies their marginal distributions. For each of the three predictions, $H_{T}$ and $H_{C}$ are independent true scores in the treatment and control condition, respectively, whereas $E_{T}$ and $E_{C}$ are corresponding measurement errors. For each prediction, the within-group variance $\sigma^{2}$ of the observed values $X_{T}$ and $X_{C}$ is partitioned into the true-score variance $\sigma_{\text {diff }}^{2}$ and the error variance $\sigma_{\text {error }}^{2}$.

partitioned into two parts, the variance $\sigma_{\text {diff }}^{2}$ due to true individual differences and the variance $\sigma_{\text {error }}^{2}$ due to unsystematic measurement errors.

The variance decomposition in D\&R's framework allows scholars to easily specify the assumed amount of heterogeneity between individuals. Instead of requiring assumptions about both the variance of true scores $\left(\sigma_{\text {diff }}^{2}\right)$ and the variance of errors $\left(\sigma_{\text {error }}^{2}\right)$, scholars can simply specify the proportion $q$ of variance due to systematic individual differences defined as $q=\sigma_{\text {diff }}^{2} / \sigma^{2}$. From the perspective of classical test theory (e.g., Revelle \& Condon, 2019), $q$ is a measure of reliability as it is the proportion of true-score variance within each condition. In the context of theory evaluation, the proportion $q$ is usually not known since it cannot be estimated in between-subjects designs based on aggregate statistics such as Cohen's $d$. Nevertheless, researchers may be able to make an informed guess about the proportion of variance due to individual differences in a specific domain. 
Assuming that all predicted effects are in the positive direction, the marginal model in Figure 1 implies that $p_{i}$ equals the probability of true individual effects being larger than zero (i.e., $H_{T, i}-H_{C, i}>0$ ). In a first step, this probability can easily be computed for each prediction when assuming that individual effects are normally distributed. Note that the proportion $p_{i}$ of individuals described by the $i^{\text {th }}$ prediction decreases as the variance of systematic individual differences increases (i.e., when assuming a larger value of the proportion $q$ ). In a second step, D\&R used the model-based estimates of $p_{i}$ as input for Equation (1) to obtain the upper and lower bounds on the proportion $p$ of individuals described by all predictions of a theory. It follows that the proportion $p$ also decreases for larger values of $q$. Importantly, however, this two-step approach does not make any assumptions about the joint distribution of individual differences across predictions. The upper and lower bounds on $p$ are merely implied by extreme cases of stochastic dependence which may not fit in with a theory. Often, psychological theories make specific assumptions about the dependence of individual differences across predictions. For instance, when assuming a common underlying trait, it follows that individual differences are correlated across predictions. D\&R's modeling approach does not allow scholars to incorporate such assumptions about the consistency of individual differences across predictions. As a remedy, I extend D\&R's framework by modeling the joint distribution of individual effects across predictions.

\section{Effect-Coded True-Score Decomposition}

Before discussing the multivariate modeling approach, it is worthwhile to reconsider the definition of the true-score variables in D\&R's univariate model in Figure 1. For each prediction, D\&R assumed that the true individual values in the treatment and the control condition $\left(H_{T}\right.$ and $H_{C}$, respectively) are independent. Often, however, this assumption is rather implausible. As an illustration, let us consider D\&R's empirical example of story spoilers (Leavitt \& Christenfeld, 2011) where the dependent variable $X$ was a rating for enjoyment of reading a story from a specific genre (e.g., a mystery story). The assumption of independent true scores implies that an individual's 
true enjoyment of reading a spoiled version of a story is independent of the enjoyment of reading an unspoiled version. This is implausible because individuals will likely differ in their general preference for a specific genre. For instance, some people will generally like mystery stories irrespective whether they are spoiled or not, whereas others generally dislike such stories. In such a case, the true scores $H_{T}$ and $H_{C}$ in each condition are not independent as assumed by D\&R's true-score model. As a remedy, I introduce a modified model that distinguishes between two sources of true individual differences, namely, an individual's general preference for a genre versus an individual's true effect of preferring the spoiled over the non-spoiled story.

The gray panels in Figure 2 show the modified decomposition which partitions each of the true scores in the treatment and control condition into two parts. I refer to this approach as "effect-coded true-score decomposition" because it distinguishes between the two parameters $\eta$, the general response level of an individual on the dependent variable, and $\delta$, the true individual effect defined as the difference between the treatment and control condition (see Appendix for details). Using the terminology of mixed-effects models (Pinheiro \& Bates, 2000), $\eta$ can be interpreted as a random intercept of individuals whereas $\delta$ is a random slope of individual treatment effects. For each prediction, the model implies that the within-group variance $\sigma^{2}$ of the observed values is decomposed into three instead of two parts: First, the variance due to true individual differences in the intercept $\left(\sigma_{\text {int }}^{2}\right)$, second, the variance due to true individual effects $\left(1 / 4 \cdot \sigma_{\text {effect }}^{2}\right.$, where the factor $1 / 4$ accounts for the scale of the parameter $\left.\delta\right)$, and third, the error variance of the dependent variable $\left(\sigma_{\text {error }}^{2}\right)$. The Appendix shows that the effect-coded true-score decomposition can be adopted both for D\&R's marginal univariate model and for the multivariate model discussed below.

The effect-coded true-score decomposition suggests a more intuitive definition and interpretation of D\&R's notion of "the proportion of variance due to individual differences." On the one hand, D\&R's true-score model defines $q$ as the proportion of variance due to any true individual differences within each condition. On the other hand, the effect-coded true-score decomposition defines $q^{*}$ as the proportion of variance due to 


\section{Figure 2}

Multivariate Normal Model of Individual Effects.

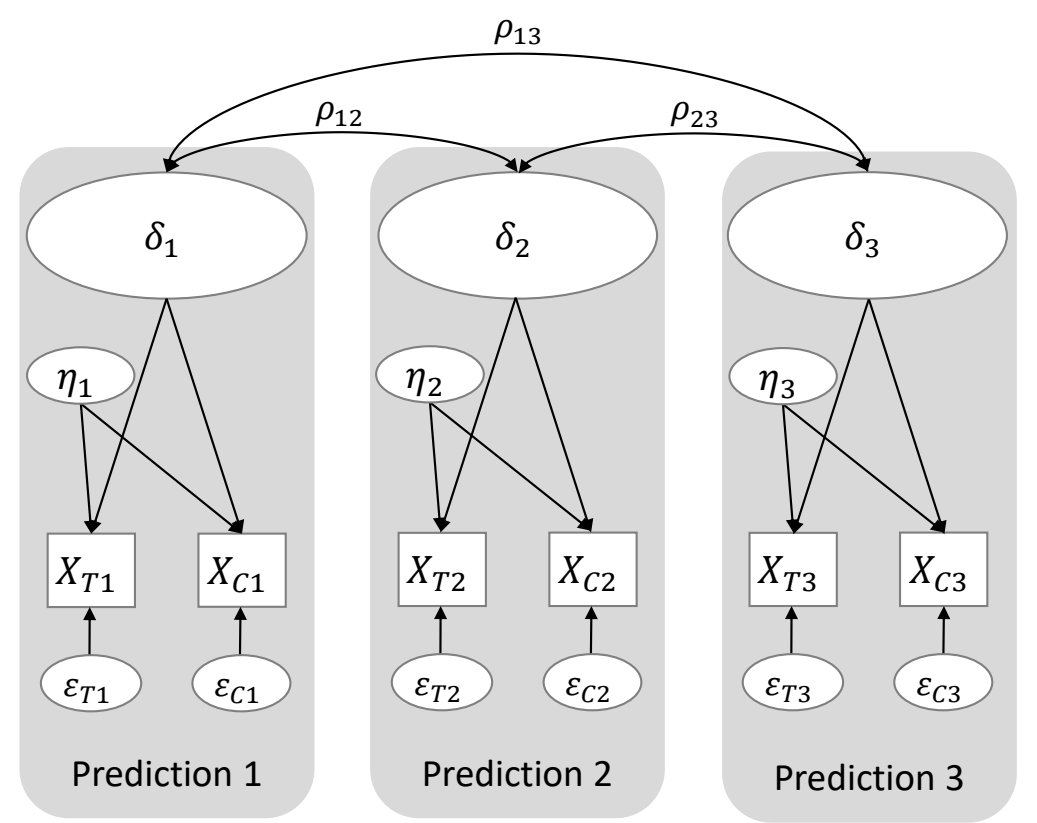

Note. The model assumes that the true individual effects $\delta$ follow a multivariate normal distribution and are correlated across predictions (parameters $\rho_{i j}$ ). For each prediction, the effect-coded true-score decomposition defines $\delta$ as the true individual difference between treatment and control condition and $\eta$ as the intercept of an individual on the dependent variable. Moreover, $\varepsilon_{T}$ and $\varepsilon_{C}$ are unsystematic measurement errors. The within-group variance $\sigma^{2}$ of the observed values $X_{T}$ and $X_{C}$ is partitioned into variance due to true individual differences in the intercept $\left(\sigma_{\text {int }}^{2}\right)$, true individual effects $\left(1 / 4 \cdot \sigma_{\text {effect }}^{2}\right)$, and measurement error $\left(\sigma_{\text {error }}^{2}\right.$; see Appendix for details).

true individual effects between the two conditions. When evaluating psychological theories, it is plausible to assume a smaller value for $q^{*}$ than for $q$ as the former refers to a smaller fraction of the true-score variance. In fact, the proportion $q$ is the maximum that can be assumed for the proportion $q^{*}$. Moreover, if a theory assumes that less than half of the variance between individuals is due to differences in the effect between conditions (the random slopes), this implies $q^{*}<q / 2$. Note that the two true-score models imply identical upper and lower bounds on the proportion $p$ when assuming $q^{*}=q / 2$ (see Appendix)

These considerations show that the choice between D\&R's true-score model and the effect-coded true-score decomposition is orthogonal to the issue of whether to model the multivariate or the marginal distribution of individual effects. However, the proportion $q^{*}$ is better suited for making assumptions about the amount of heterogeneity 
assumed by a psychological theory because theories usually focus on the existence, direction, and size of effects (i.e., the contrast between conditions). To make this specific, the theory about story spoilers by Leavitt and Christenfeld (2011) states that some individuals prefer spoiled over non-spoiled stories. However, it is not of interest whether individuals differ in their general preferences for a specific genre (e.g., whether individuals generally like or dislike crime stories). Given that most psychological theories focus on contrasts between conditions, the following considerations rely on the effect-coded true-score decomposition, and hence, the results depend on assumptions about the proportion $q^{*}$.

\section{Multivariate Normal Model of Individual Effects}

The multivariate normal model specifies the joint distribution of individual differences across all predictions of a theory instead of modeling only the marginal distributions in isolation. Figure 2 shows the multivariate model of individual effects which accounts for all predictions jointly. The vector $\boldsymbol{\delta}=\left(\delta_{1}, \ldots, \delta_{k}\right)^{T}$ refers to the true effects of an individual for the $k$ predictions of a theory. For instance, in D\&R's example "story spoilers don't spoil stories" (Leavitt \& Christenfeld, 2011), the parameter $\delta_{i}$ refers to the true preference of an individual for spoiled versus non-spoiled stories in the $i^{\text {th }}$ genre (e.g., mysteries). If all predicted effects are in the positive direction, the proportion of individuals described by all predictions of a theory is defined as the probability that all components of the random vector $\boldsymbol{\delta}$ are larger than zero (i.e., $\delta_{i}>0$ for all $i=1, \ldots, k$ ). Note that it is no longer necessary to rely on D\&R's two-step approach of first computing the proportion $p_{i}$ for each prediction separately and then applying the upper and lower bounds in Equation (1). Instead, by modeling all predictions jointly, we can directly compute the proportion $p$-at least, if we are willing to make additional assumptions about the multivariate distribution of individual effects.

In psychometrics, scholars often assume multivariate normality to account for the stochastic dependence of latent psychological constructs (e.g., in mixed-effects models and structural equation models; Kline, 2015). In line with this tradition, I assume that 
the vector $\boldsymbol{\delta}$ of (unstandardized) individual effects follows a multivariate normal distribution at the population level. Hence, the standardized individual effects also follow a multivariate normal distribution (see Appendix for details). The mean of the latter distribution is the vector of average population effect sizes (i.e., Cohen's $d$ ) for the predictions of a theory. Moreover, the covariance matrix can be decomposed into two matrices, one specifying the variances and a second specifying the correlations of individual effects. The variance of the standardized individual effects only depends on $q^{*}$, the proportion of variance due to true individual effects in the effect-coded true-score decomposition. For simplicity, I follow D\&R in assuming that the proportion $q^{*}$ is constant across predictions. Scholars can thus specify the assumed level of heterogeneity between individuals by choosing a specific value of the proportion $q^{*}$ (similar to making assumptions about the proportion $q$ in D\&R's true-score model). The correlation matrix $\boldsymbol{R}$ defines the pairwise correlations $\rho_{i j}$ of true individual effects across predictions.

Thereby, scholars can specify theoretical assumptions about the consistency of individual effects by adjusting the structure and the values of $\boldsymbol{R}$.

Figure 3 illustrates the distribution of true individual effects implied by the multivariate normal model for two predictions. In all the three panels, I assume that the true population effect sizes (i.e., Cohen's $d$ ) are $d_{1}=0.20$ and $d_{2}=0.35$ (shown by black points). Moreover, I assume that the proportion of variance due to individual effects is $q^{*}=.15$. The proportion $p$ of individuals described by the theory equals the probability that both components of the random vector $\boldsymbol{\delta}$ are positive. In Figure 3, this is the probability that individual effects fall into the upper right quadrant (indicated by the gray area). This joint probability can easily be evaluated using the cumulative distribution function of the multivariate normal distribution which is implemented in standard statistical software (Nomura, 2016).

If the correlation is $\rho=0$ (left panel in Figure 3), individual effects are independent and the proportion of individuals described by both predictions is $p=.41$. Due to independence, the proportion $p$ equals the product of the marginal proportions $p_{i}$ (i.e., the proportion of individuals described by the $i^{\text {th }}$ prediction) obtained from D\&R's 


\section{Figure 3}

Multivariate Normal Distribution of Individual Effects.
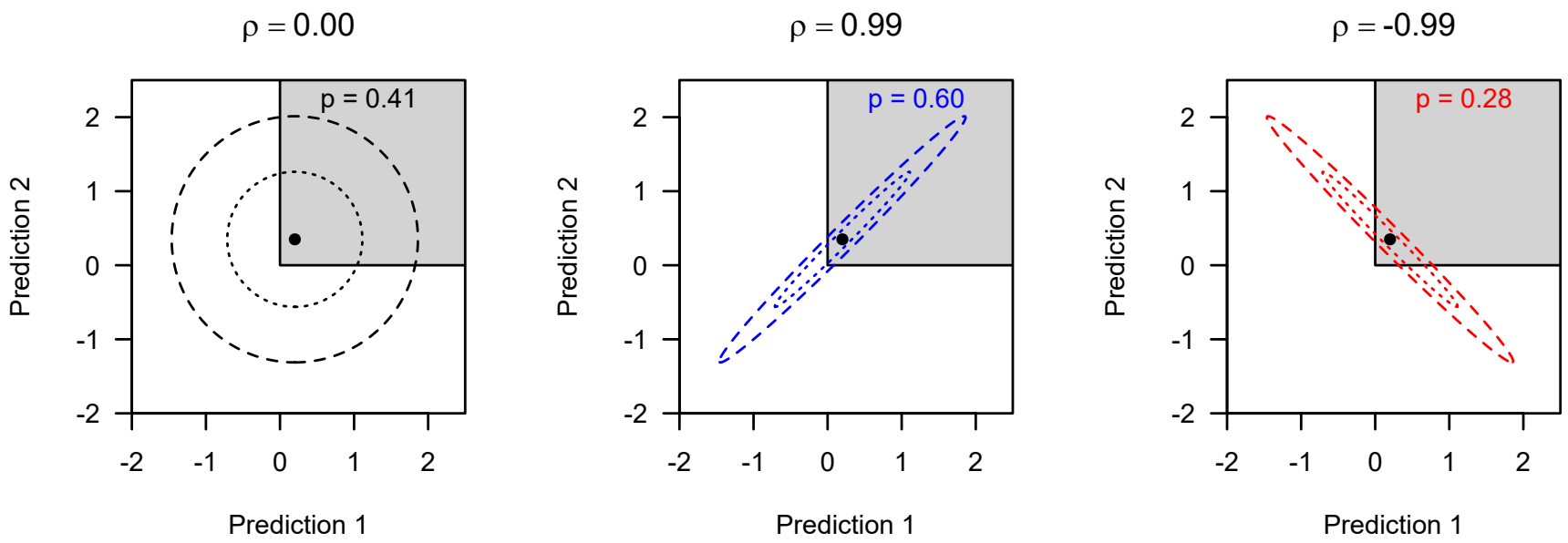

Note. The multivariate normal distribution assumes that the average population effect sizes (Cohen's $d$ ) are $d_{1}=0.20$ and $d_{2}=0.35$ (black point) and that the proportion of variance due to true individual effects is $q^{*}=.15$. The dotted and dashed ellipses show standardized individual effects covering $50 \%$ and $90 \%$ of the population, respectively. The proportion $p$ of individuals satisfying both of two predictions is shown by the upper right quadrant in gray.

univariate normal model,

$$
p=\prod_{i=1}^{k} p_{i} .
$$

If the correlation is close to perfect ( $\rho=.99$, middle panel in Figure 3), the proportion of individuals described by all predictions increases to $p=.60$. If the correlation is strongly negative $(\rho=-.99$, right panel), the proportion $p$ decreases to $p=.28$. These two cases (more precisely, $\rho=1$ and $\rho=-1$ ) define the upper and lower bounds implied by the multivariate model. Notably, these bounds are identical to D\&R's upper and lower bounds even though the latter only assume normality of the two marginal distributions but not of the joint distribution. This is due to the fact that the individual effects from the bivariate normal distribution have a perfect positive and negative dependence if $\rho=1$ and $\rho=-1$, respectively. ${ }^{1}$ Below, I show that the upper and lower bounds implied by the two approaches are identical only if $k=2$ and that D\&R's lower bound is strictly below the multivariate lower bound if a theory makes at least $k \geq 3$ predictions.

If a theory makes more than two predictions, it may be difficult to specify the full

\footnotetext{
${ }^{1}$ In probability theory, perfect positive dependence is referred to as comonotonicity of random variables. The opposite concept of countermonotonicity is only defined in $k=2$ dimensions (Lee \& Ahn, 2014).
} 
matrix $\boldsymbol{R}$. This would require scholars to make specific assumptions about all $k(k+1) / 2$ pairwise correlations of individual effects for the $k$ predictions. As a remedy, I define a constrained model version which assumes that all pairwise correlations of individual effects are equal, $\rho_{i j}=\rho$. For instance, for $k=3$ predictions, the correlation matrix thus becomes

$$
\boldsymbol{R}=\left(\begin{array}{lll}
1 & \rho & \rho \\
\rho & 1 & \rho \\
\rho & \rho & 1
\end{array}\right)
$$

The parameter $\rho$ has an intuitive interpretation in terms of the overall level of consistency of individual effects across predictions.

The constrained model version allows scholars to specify the assumed level of consistency of individual effects by defining a single parameter as opposed to the full matrix $\boldsymbol{R}$. For instance, assuming $\rho=1$ resembles perfect linear dependence of individual effects across predictions. This is equivalent to assuming a single, unidimensional trait across predictions meaning that the latent variables $\delta_{i}$ in Figure 2 collapse into a single latent factor. In the example of story spoilers, such a trait would imply that individual preferences for spoiled vs. non-spoiled stories are perfectly consistent across different genres. In contrast, the case $\rho=0$ is equivalent to assuming stochastic independence of individual effects across predictions. ${ }^{2}$ In the example of story spoilers, this would imply that individual preferences for spoilers in one genre are not related to individual preferences for spoilers in another genre.

Between these two extremes of perfect dependence and independence, assuming $0<\rho<1$ is equivalent to a higher-order factor underlying individual effects across predictions (Yung et al., 1999). This means that the individual effects $\delta_{i}$ in Figure 2 share a common factor but still have true-score variance unique to each prediction. In the example of story spoilers, individuals would thus have a general preference for or against spoilers that may still differ depending on the specific genre. The assumption of a higher-order factor underlying individuals effects (i.e., $0<\rho<1$ ) is also plausible for the

\footnotetext{
${ }^{2}$ In general, a correlation coefficient of zero does not imply statistical independence - this is only true for the multivariate normal distribution.
} 
remaining three empirical examples by D\&R. First, the degree to which text comprehension is compromised by interruptions during reading (Foroughi et al., 2015) may reflect stable individual differences in working memory. Second, the degree to which personal predictions about the future are optimistically biased (Armor et al., 2008) may reflect subjective values and beliefs. Third, the degree to which moral judgments are focused on the evaluation of the acting person as opposed to an act itself (Schweinsberg et al., 2016; Uhlmann et al., 2015) may be due to stable differences in moral reasoning.

\section{Comparison of Upper and Lower Bounds}

Figure 4 compares D\&R's upper and lower bounds (i.e., the top and bottom fringes of the gray area, respectively) to the upper and lower bounds implied by the multivariate normal model (the blue and red curve, respectively). ${ }^{3}$ The five curves illustrate the proportion $p$ of individuals described by all predictions of a theory (y-axis) for different levels of consistency. Perfect dependence is modeled by a pairwise correlation of $\rho=1$; high and medium levels of consistency are modeled by $\rho=.80$ and $\rho=.50$, respectively; and independence is modeled by $\rho=0$. Negative stochastic dependence is modeled by a negative pairwise correlation $\rho$ with the exact value depending on the number of predictions in each example.

Irrespective of the assumed correlation, the proportion $p$ decreases monotonically as a function of the proportion $q^{*}$ of variance due to true individual effects. ${ }^{4}$ Moreover, the multivariate normal model shows that the proportion $p$ increases as individual effects become more consistent across predictions (i.e., if $\rho$ increases). When assuming perfect stochastic dependence $(\rho=1)$, the proportion $p$ is identical to D\&R's upper bound. In this case, true individual effects for each prediction are fully explained by a common, underlying trait. As the predictions of a theory are usually domain-specific, the assumption of a single underlying trait may often be overly strong, meaning that scholars

\footnotetext{
${ }^{3}$ An R Shiny web application available at https://dheck.shinyapps.io/paradox-converging-evidence/ allows readers to generate plots similar to that in Figure 4 for a given set of effect sizes. The R code for replicating all analyses is available at the OSF repository (Heck, 2021).

${ }^{4}$ The $\mathrm{x}$-axis in Figure 4 is restricted to $q^{*} \in[0, .50]$ to provide plots comparable to D\&R's plots showing $q \in[0,1]$ (cf. Secion Effect-Coded True-Score Decomposition).
} 


\section{Figure 4}

Proportion $p$ of Individuals Described by All Predictions of a Theory.

(A) Leavitt \& Christenfeld (2011)

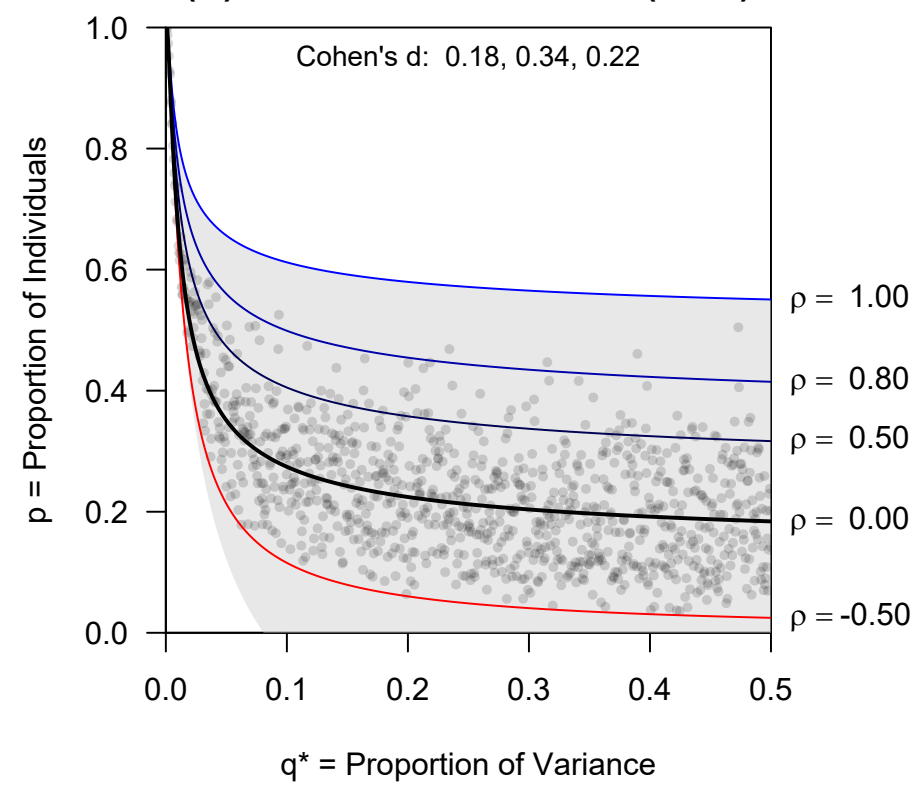

(C) Armor et al. (2008)

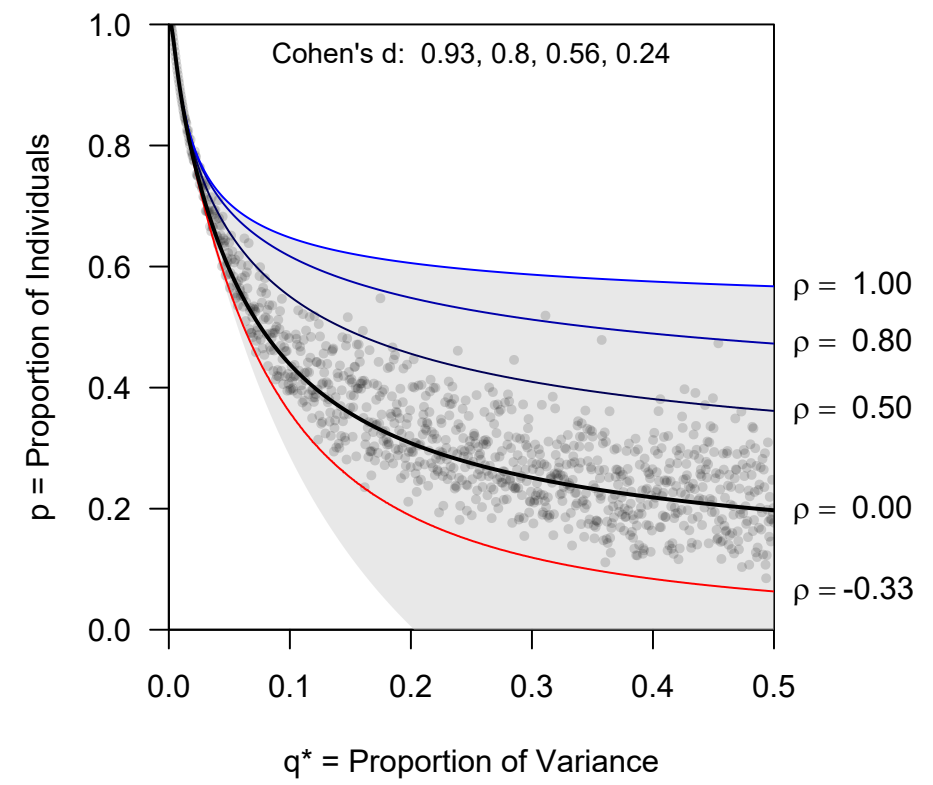

(B) Foroughi et al. (2015)

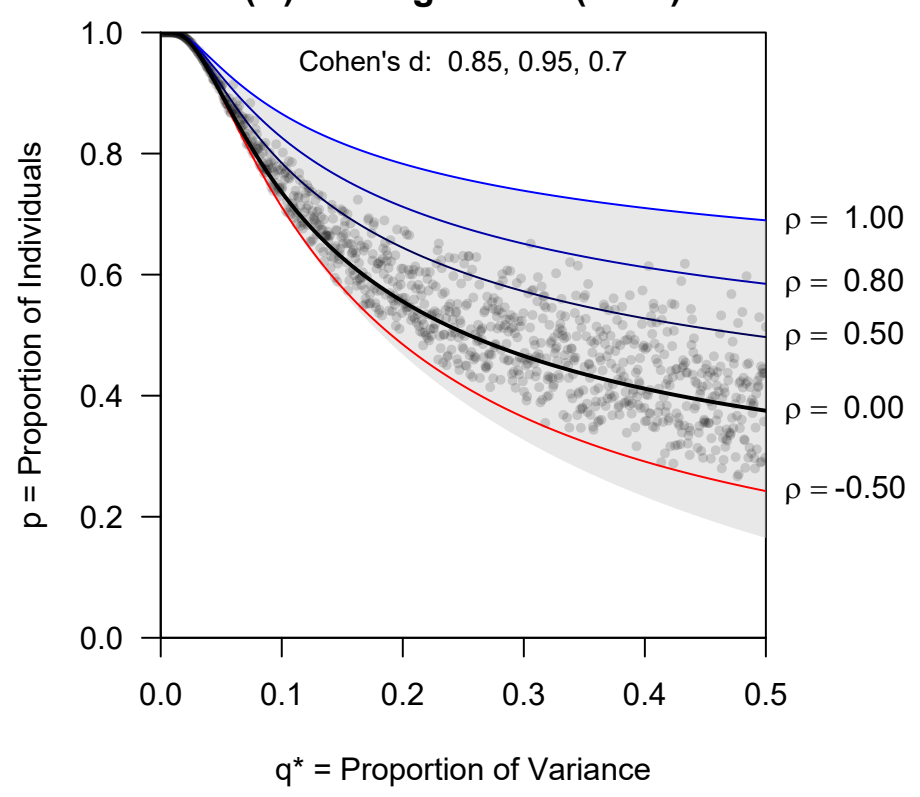

(D) Schweinsberg et al. (2016)

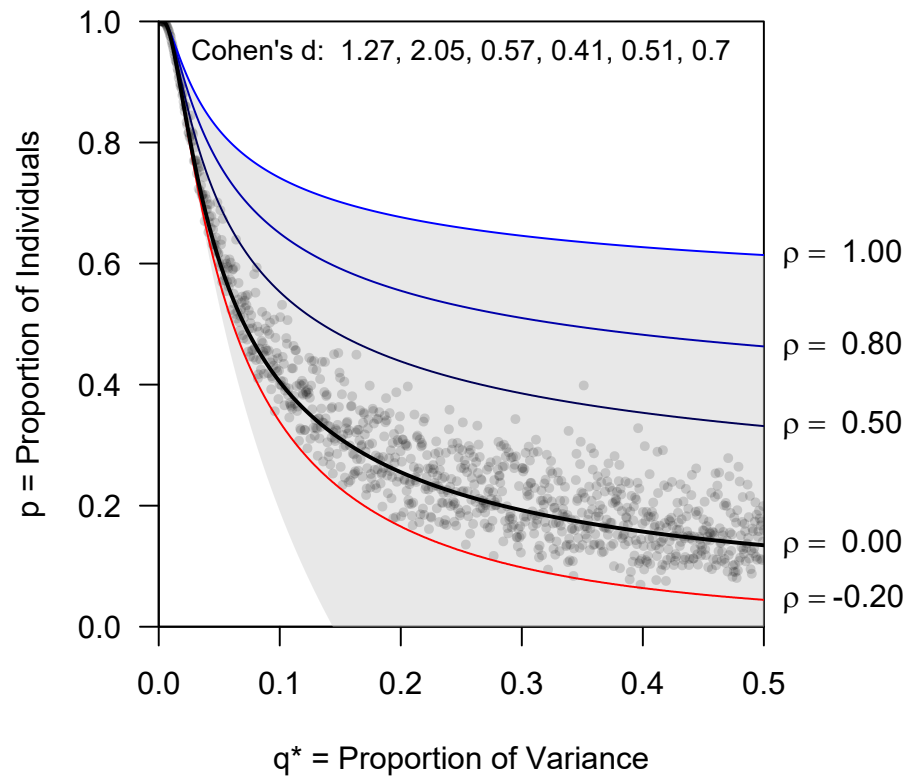

Note. On the x-axis, $q^{*}$ is the proportion of variance due to true individual effects. On the $\mathrm{y}$-axis, $p$ is the proportion of individuals described by all predictions of a theory. The top and bottom fringes of the gray area are D\&R's upper and lower bounds on $p$, respectively. The five solid lines are based on the assumption that all pairwise correlations of individual effects in the multivariate normal model are equal to $\rho$. Gray points show specific values of $p$ for correlation matrices $\boldsymbol{R}$ randomly sampled from a uniform distribution. 
should be careful when interpreting the upper bound on the proportion $p$. When assuming a very large positive correlation $(\rho=.80)$, the proportion $p$ is still close to the upper bound. In this scenario, a theory assumes that true individual effects share a common, higher-order trait across predictions while still having true-score variance unique for each prediction (Yung et al., 1999). When assuming a lower level of consistency $(\rho=.50)$, the proportion $p$ decreases substantially even though this still resembles a 'large' correlation according to the conventions by Cohen (1988). Another theoretically interesting scenario is the assumption of independence $(\rho=0)$, meaning that individual differences are context-specific for each prediction and do not share any common variance (similar to distinct states; Steyer et al., 2015). In this case, the proportion $p$ is closer to the lower bound than to the upper bound.

Figure 4 also shows the proportion $p$ implied by assuming a negative pairwise correlation $\rho$ of individual effects across predictions. Such a negative dependence is difficult to interpret substantively since it implies statements of the form "the larger effect A, the smaller effect B" for all pairs of predictions. Compared to assuming positive dependence or independence, the case $\rho<0$ is thus less relevant for psychological theories. However, by assessing the most extreme negative correlation possible, we will see that the lower bound for $p$ implied by the multivariate normal model is strictly above the D\&R's lower bound if a theory makes at least $k \geq 3$ predictions.

To derive the lower bound implied by the multivariate normal model, we can draw on the fact that the proportion $p$ of individuals for whom the theory holds is maximized if $\rho=1$ and minimized if $\rho=-1 /(k-1)$ (Kotz \& Seeger, 1992, Remark 2). In the latter case, the pairwise correlation assumes the lowest possible value that still ensures that $\boldsymbol{R}$ is a valid correlation matrix (i.e., a positive semidefinite matrix). ${ }^{5}$ The proportion $p$ obtained by assuming $\rho=-1 /(k-1)$ is thus the lower bound implied by the multivariate normal distribution. Importantly, even when loosening the assumption that all correlations are equal, it is impossible to construct a correlation matrix that results in a

\footnotetext{
${ }^{5}$ For $\rho=-1 /(k-1)$, the $k$-dimensional normal distribution reduces to a $(k-1)$-dimensional normal distribution on a lower-dimensional hyperplane $\left\{\boldsymbol{\delta} \mid \sum \delta_{i}=0\right\}$ which is perpendicular to $\left\{\boldsymbol{\delta} \mid \delta_{1}=\cdots=\delta_{k}\right\}$ (Kotz \& Seeger, 1992). As illustrated in Figure 5, this means that the case $\rho=-1 /(k-1)$ is orthogonal to assuming perfect consistency of individual differences (i.e., $\rho=1$ ).
} 
multivariate normal distribution with a proportion $p$ smaller than this lower bound (Kotz \& Seeger, 1992, Lemma 1). Figure 4 illustrates this lower bound, for instance, using $\rho=-1 / 2$ for $k=3$ predictions (Leavitt \& Christenfeld, 2011) and using $\rho=-1 / 3$ for $k=4$ predictions (Armor et al., 2008).

If a theory makes at least $k=3$ predictions, the lower bound of the multivariate normal is strictly above D\&R's lower bound. This is due to the fact that, in order to move closer to D\&R's lower bound, one may construct non-normal joint distributions as long as the marginals are univariate normal distributions (e.g., using copulas; Colonius, 2016). More generally, when $k \geq 3$, there does not exist any multivariate probability distribution that implies the equality of the proportion $p$ and Fréchet's lower bound in Equation (1) (Lee \& Ahn, 2014; Lee et al., 2017). ${ }^{6}$ This means that, even when constructing other joint distributions than the multivariate normal, D\&R's lower bound cannot be interpreted as a possible 'worst-case scenario' because it does not correspond to any logically admissible joint distribution of individual effects across predictions. As a remedy, we could aim at finding a joint distribution that approximates D\&R's lower bound from above. However, minimizing the distance to the lower bound requires strong structural constraints on the joint distribution (i.e., exchangeability of the copula; Kotz \& Seeger, 1992) which may be hard to justify based on a psychological theory. Overall, constructing a joint distribution that approximates the lower bound is a nontrivial endeavor, especially since probability theory does not provide a unique definition of extreme negative dependence for more than two random variables (Puccetti \& Wang, 2015).

The assumptions underlying D\&R's lower bound are not very intuitive for specifying individual differences in psychological theories. Often, a multivariate normal distribution may be a more suitable choice for specifying assumptions about the dependence of psychological constructs in a substantive theory. However, even when assuming that the joint distribution is multivariate normal, the corresponding lower bound is only obtained when all individual effects show negative pairwise correlations.

\footnotetext{
${ }^{6}$ As remarked by a reviewer, the equality can be achieved for $k \geq 3$ point-wise. By fixing a set of values, a copula can be constructed to attain the lower bound given a set of marginal distribution functions. However, the resulting structure would not be a joint distribution function of all random variables.
} 


\section{Figure 5}

Three-Dimensional Normal Distribution of Individual Effects.
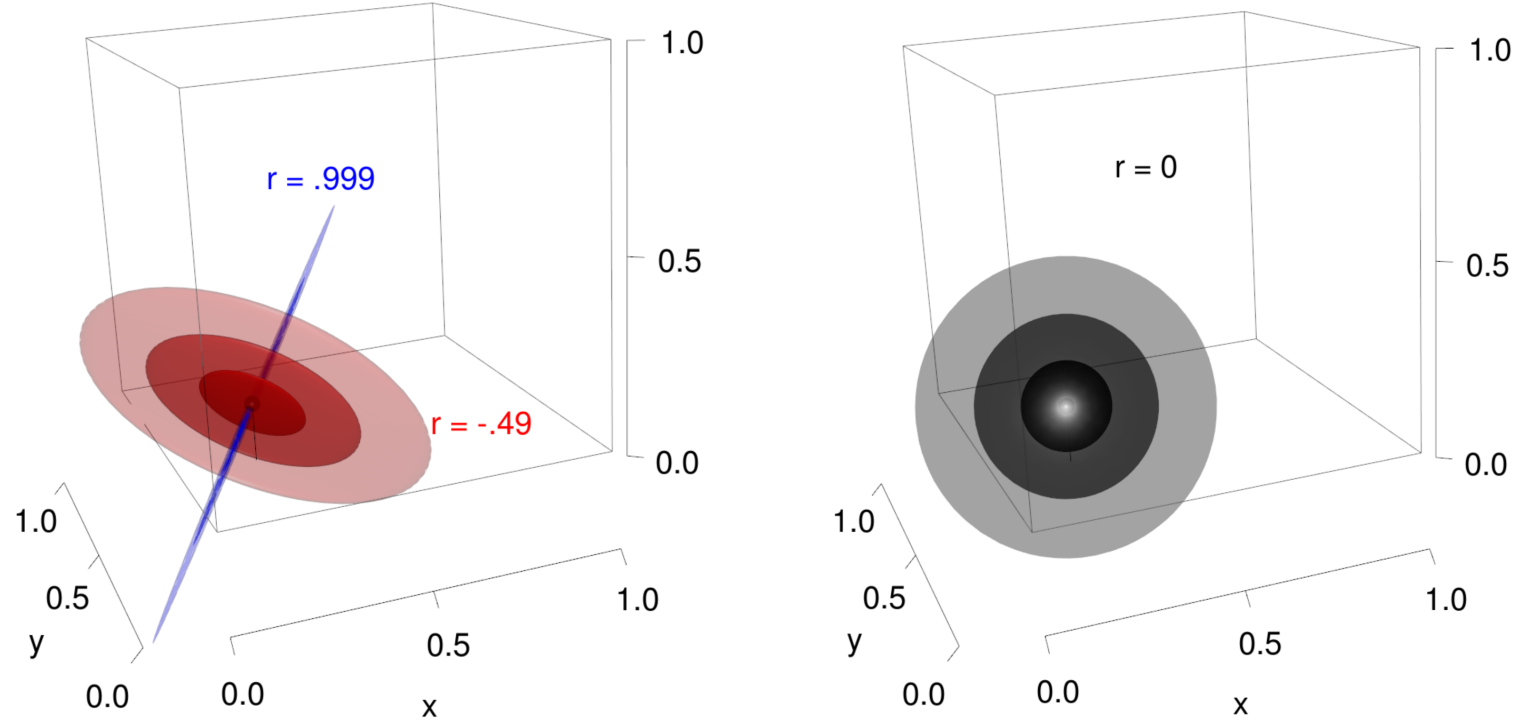

Note. The 3-dimensional normal distribution assumes that the average population effect sizes (Cohen's $d$ ) for the three predictions are $d_{1}=0.20, d_{2}=0.35$, and $d_{2}=0.15$ and that the proportion of variance due to true individual effects is $q^{*}=.15$. The ellipses show standardized individual effects covering 10\%, 50\%, and $90 \%$ of the population, respectively. Individual effects inside the box in the upper right quadrant are in line with all three predictions of the theory.

Figure 5 illustrates the joint distribution for $k=3$ dimensions. When assuming (nearly) perfect positive dependence $(\rho=.999)$, the multivariate distribution collapses into a single dimension which represents a common trait for all predictions of a theory. In contrast, when assuming (nearly) maximum negative dependence $(\rho=-.49)$, the multivariate distribution collapses to a 2-dimensional distribution of individual effects on a plane orthogonal to the common-trait dimension (see Footnote 5; Kotz \& Seeger, 1992). It is difficult to imagine a theory that predicts such a complex structure of individual effects across predictions. For instance, in the case of story spoilers, this would mean that, for all pairwise combinations of genres, the more individuals prefer spoilers in one kind of genre, the less they prefer spoilers in another genre.

As a remedy, the assumption that individual differences are independent $(\rho=0)$ may provide a more appropriate 'worst-case scenario' for evaluating the scope of many psychological theories. Figure 5 shows that the corresponding joint distribution is a symmetric sphere centered at the vector of average effect sizes at the population level. This means that the predictions of a theory refer to unrelated, context-specific 
psychological constructs (similar to different states). In general, it depends on a given theory whether the assumption of independence or negative dependence is a more suitable worst-case assumption. Moreover, even when committing to the stronger assumption of independence, Figure 4 shows that the resulting proportion $p$ can still be quite low, meaning that a theory may describe only a minority of the population.

So far, I assumed that all pairwise correlations in the multivariate normal model in Figure 2 are equal to $\rho$. This simplifying constraint is useful to facilitate an intuitive interpretation of different levels of consistency of individual effects but may be overly restrictive in practice. As a remedy, the gray points in Figure 4 show specific values of the proportion $p$ when assuming arbitrary, unstructured correlation matrices $\boldsymbol{R}$ randomly sampled from a uniform distribution. Note that these values must be between the lower and the upper bound derived for the multivariate normal distribution (Kotz \& Seeger, 1992). Figure 4 shows that most of the sampled proportions fall into the area between the curves for the correlation $\rho=.50$ and the lowest possible correlation $\rho=-1 /(k-1)$. Hence, it is not sufficient to merely assume a multivariate normal distribution with any type of correlation matrix in order to obtain a proportion $p$ close to the upper bound. The proportion $p$ is only maximized if one assumes that individual effects are highly correlated across predictions.

\section{Discussion}

The present comment extends Davis-Stober and Regenwetter's (2019) framework for modeling the proportion $p$ of individuals described by all predictions of a theory. Whereas D\&R's model only makes assumptions about the marginal distribution of individual effects for each prediction, the multivariate normal model accounts for their joint distribution. This facilitates a more detailed assessment of the proportion $p$ of individuals described by all predictions of a theory without applying Fréchet's upper and lower bounds in Equation (1). Moreover, in contrast to D\&R's original approach, the effect-coded true-score decomposition partitions individual differences within the treatment and control condition into true individual effects (random slopes) and true 
individual differences in the overall level on the dependent variable (random intercepts; see Figure 2). To apply the model, it is necessary to make theoretical assumptions about the variance of true individual effects (i.e., the proportion $q^{*}$ ) and the level of consistency (i.e., the pairwise correlation $\rho$ ).

The joint modeling approach offers the advantage of allowing scholars to specify the assumed level of consistency of individual effects across predictions. For instance, in D\&R's example of story spoilers (Leavitt \& Christenfeld, 2011), the precision of the theory could be improved by specifying whether individual preferences for spoiled vs. non-spoiled stories are assumed to be consistent across different genres. If such an assumption is plausible substantively, this corresponds to a perfect or very high correlation of individual effects (e.g., $\rho \geq .80$ ). Assuming a strong positive dependence of true individual effects across predictions maximizes the proportion $p$ of individuals described by all predictions of a theory, thus increasing its scope. Besides modeling the joint distribution, the effect-coded true-score decomposition allows scholars to specify whether most of the variance between individuals is due to differences in the effect between conditions (i.e., $q^{*}>q / 2$ ) or due to differences in the overall level on the dependent variable (i.e., $q^{*}<q / 2$; see Effect-Coded True-Score Decomposition). In the latter case, the proportion $p$ of individuals described by a theory is larger compared to that of D\&R's original approach, thus further increasing the scope of a theory.

However, assuming a high consistency $(\rho)$ and a small variance $\left(q^{*}\right)$ of individual effects cannot fully resolve the 'paradox' of converging evidence. The proportion $p$ in the multivariate normal model is bounded from above by D\&R's upper bound, and hence, including additional predictions can only result in a decrease of the proportion of individuals described by a theory. As a remedy, Davis-Stober and Regenwetter (2019, p. 877) discussed several solutions of how to avoid the 'paradox' entirely, for instance, by restricting the scope a theory to a subpopulation for which it holds without exception, by constraining all individual effects to be in the predicted direction, or by assuming that the theory holds for all individuals probabilistically (see also Bredenkamp, 1980; Popper, 2002). In cases where these solutions are not feasible (e.g., because individual differences 
are central to a theory), the multivariate normal model provides a useful theoretical tool to specify a joint distribution of individual effects and to assess the consequences of such assumptions for the scope of a theory.

The multivariate normal model implies a lower bound on the proportion $p$ that is above the lower bound derived by $\mathrm{D} \& \mathrm{R}$ based on the marginal distributions only. The lower bound of the multivariate normal assumes that individual effects are negatively correlated for all pairwise combinations of predictions. As illustrated in Figure 5, this is a rather unintuitive assumption unsuitable for modeling the joint distribution of many psychological constructs. The lower bound assuming extreme negative dependence may thus be of limited relevance in practice. As a remedy, stochastic independence of individual effects across predictions $(\rho=0)$ will often provide a more suitable 'worst-case scenario' for evaluating the scope of psychological theories. Assuming independence also offers the benefit that scholars do not have to assume that the joint distribution of individual effects is multivariate normal since this follows necessarily if the marginal distributions are univariate normal (Flury, 1997, p. 176). Figure 4 shows that the independence assumption results in a proportion $p$ of individuals described by all predictions of a theory that is clearly above D\&R's lower bound. Nevertheless, the proportion $p$ is still closer to the lower bound than to the upper bound.

The present analysis adopted D\&R's conceptual approach of merely assuming a certain proportion of variance due to true individual effects and a corresponding correlation structure. Alternatively, it is possible to estimate the variance of individual effects and their correlation across predictions by fitting the multivariate model in Figure 2 to data. This requires to test all predictions for all individuals using a within-subjects design in which individuals provide multiple responses both in the treatment and in the control condition (multiple responses are necessary to ensure identifiability of the individual effects; Pinheiro \& Bates, 2000). Statistically, latent variable modeling can then be applied to assess the reliability of the individual effects by estimating the proportion $q^{*}$ of true-score variance in Figure 2 (e.g., Gollwitzer et al., 2014). One can also estimate the consistency of individual effects (i.e., the matrix $\boldsymbol{R}$ ) if 
the predictions of a theory are jointly tested in a within-subjects design (cf. D\&R, p. 876). For this purpose, scholars may adopt advanced psychometric models such as latent state-trait theory (Steyer et al., 2015) to partition the true-score variance into a prediction-specific and a general part.

Overall, I agree with D\&R's conclusion that scholars need to "explicitly state who the theory applies to and whether the predicted 'feature' in question is a probabilistic outcome" (p. 878). However, there is another important aspect of psychological theories scholars should pay attention to: If a theory assumes the existence of true individual differences, it is necessary to specify whether individual effects show a certain level of consistency across predictions (similar to a trait) or whether they are statistically independent (similar to a state). For this purpose, researchers can draw on methods and models that have been developed in psychometrics to account for heterogeneity between individuals. The present comment thus shows that Davis-Stober and Regenwetter were correct in their conjecture that "hierarchical models might sometimes spell out the joint distributions of multiple predictions, in which case the scholar may be able to move beyond the upper and lower bounds" (p. 877). 


\section{References}

Armor, D. A., Massey, C., \& Sackett, A. M. (2008). Prescribed optimism: Is it right to be wrong about the future? Psychological Science, 19, 329-331. https://doi.org/10.1111/j.1467-9280.2008.02089.x

Bredenkamp, J. (1980). Theorie und Planung psychologischer Experimente. Steinkopff.

Cohen, J. (1988). Statistical power analysis for the behavioral sciences (2nd ed.). Erlbaum.

Colonius, H. (2016). An invitation to coupling and copulas: With applications to multisensory modeling. Journal of Mathematical Psychology, 74, 2-10. https://doi.org/10.1016/j.jmp.2016.02.004

Davis-Stober, C. P., \& Regenwetter, M. (2019). The 'paradox' of converging evidence. Psychological Review, 126, 865-879. https://doi.org/10.1037/rev0000156

Flury, B. (1997). A first course in multivariate statistics. Springer-Verlag. https://doi.org/10.1007/978-1-4757-2765-4

Foroughi, C. K., Werner, N. E., Barragán, D., \& Boehm-Davis, D. A. (2015). Interruptions disrupt reading comprehension. Journal of Experimental Psychology: General, 144, 704-709. https://doi.org/10.1037/xge0000074

Fréchet, M. (1951). Sur les tableaux de corrélation dont les marges sont données. Annales de I'Université de Lyon. Section A: Sciences mathématiques et astronomie, 9, $53-77$.

Gollwitzer, M., Christ, O., \& Lemmer, G. (2014). Individual differences make a difference: On the use and the psychometric properties of difference scores in social psychology. European Journal of Social Psychology, 44, 673-682. https://doi.org/10.1002/ejsp.2042

Heck, D. W. (2021). Assessing the 'paradox' of converging evidence by modeling the joint distribution of individual differences: Comment on Davis-Stober and Regenwetter (2019). Supplementary material. https://osf.io/7fk49/

Kline, R. B. (2015). Principles and practice of structural equation modeling (Fourth edition). Guilford Publications. 
Kotz, S., \& Seeger, J. P. (1992). Lower bounds on multivariate distributions with preassigned marginals. Lecture Notes-Monograph Series, 22, 211-218.

Leavitt, J. D., \& Christenfeld, N. J. S. (2011). Story spoilers don't spoil stories. Psychological Science, 22, 1152-1154. https://doi.org/10.1177/0956797611417007 Lee, W., \& Ahn, J. Y. (2014). On the multidimensional extension of countermonotonicity and its applications. Insurance: Mathematics and Economics, 56, 68-79. https://doi.org/10.1016/j.insmatheco.2014.03.002

Lee, W., Cheung, K. C., \& Ahn, J. Y. (2017). Multivariate countermonotonicity and the minimal copulas. Journal of Computational and Applied Mathematics, 317, 589-602. https://doi.org/10.1016/j.cam.2016.12.032

Lord, F. M., \& Novick, M. R. (1968). Statistical theories of mental test scores. Addison-Wesley.

Nomura, N. (2016). Evaluation of Gaussian orthant probabilities based on orthogonal projections to subspaces. Statistics and Computing, 26, 187-197. https://doi.org/10.1007/s11222-014-9487-8

Pinheiro, J., \& Bates, D. (2000). Mixed-effects models in S and S-PLUS. Springer-Verlag. Popper, K. (2002). The logic of scientific discovery. Routledge. https://doi.org/10.4324/9780203994627

Puccetti, G., \& Wang, R. (2015). Extremal dependence concepts. Statistical Science, 30, 485-517. https://doi.org/10.1214/15-STS525

Revelle, W., \& Condon, D. M. (2019). Reliability from alpha to omega: A tutorial. Psychological Assessment, 31, 1395-1411. https://doi.org/10.1037/pas0000754

Schweinsberg, M., Madan, N., Vianello, M., Sommer, S. A., Jordan, J., Tierney, W., ... Uhlmann, E. L. (2016). The pipeline project: Pre-publication independent replications of a single laboratory's research pipeline. Journal of Experimental Social Psychology, 66, 55-67. https://doi.org/10.1016/j.jesp.2015.10.001

Steyer, R., Mayer, A., Geiser, C., \& Cole, D. A. (2015). A theory of states and traits-revised. Annual Review of Clinical Psychology, 11, 71-98. https://doi.org/10.1146/annurev-clinpsy-032813-153719 
Uhlmann, E. L., Pizarro, D. A., \& Diermeier, D. (2015). A person-centered approach to moral judgment. Perspectives on Psychological Science, 10, 72-81. https://doi.org/10.1177/1745691614556679

Yung, Y.-F., Thissen, D., \& McLeod, L. D. (1999). On the relationship between the higher-order factor model and the hierarchical factor model. Psychometrika, 64 , 113-128. https://doi.org/10.1007/BF02294531 


\section{Appendix}

\section{Formal Definition of the Multivariate Normal Model}

In the following, I provide a formal definition of the multivariate normal model shown in Figure 2. The model decomposes the true scores $H_{T}$ and $H_{C}$ in D\&R's model in Figure 1 into an individual's intercept on the dependent variable $(\eta)$ and the true individual effect $(\delta)$. Going beyond classical test theory, the observed values in the treatment condition are modeled as

$$
X_{T}=\underbrace{\eta+\frac{1}{2} \delta}_{H_{T}}+\varepsilon_{T} .
$$

The model equation for the control group is equivalently defined as

$$
X_{C}=\underbrace{\eta-\frac{1}{2} \delta}_{H_{C}}+\varepsilon_{C} .
$$

It follows that $\delta$ is the true individual difference between treatment and control condition and has thus a similar interpretation as the parameter $\Delta_{H}$ in D\&R's model.

The random variables on the right-hand side of Equation (A1) and (A2) are assumed to be independent. Hence, the within-group variance $\sigma^{2}=\operatorname{Var}\left(X_{T}\right)=\operatorname{Var}\left(X_{C}\right)$ can be decomposed into three parts,

$$
\begin{aligned}
\sigma^{2} & =\operatorname{Var}(\eta)+\operatorname{Var}\left(\frac{1}{2} \delta\right)+\operatorname{Var}(\varepsilon) \\
& =\underbrace{\sigma_{\text {int }}^{2}+\frac{1}{4} \sigma_{\text {effect }}^{2}}_{\sigma_{\text {diff }}^{2}}+\sigma_{\text {error }}^{2} .
\end{aligned}
$$

It follows that the proportion $q^{*}$ of variance due to true individual effects is

$$
q^{*}=\frac{1 / 4 \sigma_{\text {effect }}^{2}}{\sigma^{2}}
$$

Note that it is invalid to define the proportion of variance as $\sigma_{\text {effect }}^{2} / \sigma^{2}$ because this ratio becomes larger than one if $\sigma_{\text {effect }}^{2}>4 / 3\left(\sigma_{\text {int }}^{2}+\sigma_{\text {error }}^{2}\right)$.

We can now apply the effect-coded true-score decomposition to D\&R's marginal 
univariate model in Figure 1. To derive the proportion $p_{i}$ of individuals described by prediction $i, \mathrm{D} \& \mathrm{R}$ assumed that the true individual effects follow a univariate normal distribution,

$$
\delta \sim \mathcal{N}\left(\mu_{T}-\mu_{C}, \sigma_{\text {effect }}^{2}\right)
$$

where $\mu_{T}$ and $\mu_{C}$ are the means in the treatment and control condition, respectively. Next, the difference between treatment and control condition is standardized by the within-group standard deviation $\sigma$,

$$
\delta^{\prime} \sim \mathcal{N}\left(d, \frac{\sigma_{\text {effect }}^{2}}{\sigma^{2}}\right)
$$

where $d=\left(\mu_{T}-\mu_{C}\right) / \sigma$ is the average population effect (i.e., Cohen's $\left.d\right)$. The variance of this distribution equals $4 q^{*}$. For comparison, in D\&R's original approach, the variance of the standardized difference $\Delta_{H}$ equals $2 q$ (see p. 879). The two true-score models thus imply identical upper and lower bounds if $4 q^{*}=2 q$, that is, when specifying $q^{*}=1 / 2 q$. Hence, the proportion $q^{*}$ within the effect-coded true-score decomposition has a different interpretation and scaling than the proportion $q$ within D\&R's true-score model. Finally, we obtain the proportion $p_{i}=P(\delta>0)$ using the cumulative distribution function of the standard normal distribution,

$$
p_{i}=\Phi\left(\frac{d}{2 \sqrt{q^{*}}}\right)
$$

To model the joint distribution of individual effects across predictions, I assume a multivariate normal distribution,

$$
\boldsymbol{\delta} \sim \mathcal{M V N}\left(\boldsymbol{\mu}_{T}-\boldsymbol{\mu}_{C}, \boldsymbol{D R} \boldsymbol{D}\right)
$$

where $\boldsymbol{\mu}_{T}$ and $\boldsymbol{\mu}_{C}$ are the mean vectors for the treatment and control conditions, respectively. The covariance matrix depends on the correlation matrix $\boldsymbol{R}$ and on the diagonal matrix $\boldsymbol{D}$ which contains the individual-effect variances, $D_{i i}=\sigma_{\text {effect }, i}^{2}$ for $i=1, \ldots, k$. Similar as for the univariate model, we rescale the individual effects in Equation (A8) by the within-group standard deviations $\sigma_{1}, \ldots, \sigma_{k}$, thus obtaining the 
distribution of standardized individual effects

$$
\delta^{\prime} \sim \mathcal{M V N}(\boldsymbol{d}, Q R Q)
$$

where $\boldsymbol{d}=\left(d_{1}, \ldots, d_{k}\right)^{T}$ is the vector of average population effect sizes (i.e., Cohen's $d$ ). In contrast to the matrix $\boldsymbol{D}$, the diagonal matrix $\boldsymbol{Q}$ does not depend on the variances $\sigma_{\text {effect }, i}^{2}$ but only on the proportion $q^{*}$,

$$
Q_{i i}=\frac{\sigma_{\text {effect }, i}^{2}}{\sigma_{i}^{2}}=4 q^{*} .
$$

For the multivariate normal distribution, the proportion of the population $p$ described by all predictions (i.e., $\delta_{i}>0$ for all $i=1, \ldots, k$ ) is given by the integral

$$
p=\int_{0}^{\infty} \cdots \int_{0}^{\infty} f_{\mathcal{M V N}(\boldsymbol{d}, \boldsymbol{Q} \boldsymbol{R} \boldsymbol{Q})}\left(\left(\delta_{1}^{\prime}, \ldots, \delta_{k}^{\prime}\right)^{T}\right) \mathrm{d} \delta_{1}^{\prime} \ldots \mathrm{d} \delta_{k}^{\prime}
$$

where $f_{\mathcal{M V N}}$ denotes the probability density function of the multivariate normal distribution with mean and covariance as defined in Equation (A9). In the statistics literature, approximations are available to evaluate the multivariate normal orthant probabilities illustrated in Figure 3 (e.g., Nomura, 2016). 